\title{
MODELOS REALISTAS EN LA VERIFICACIÓN DEL ESFUERZO CORTANTE EN ELEMENTOS DE HORMIGÓN ARMADO
}

\section{Francisco Aguirre, Sebastián Ontiveros y Alejandra Orellana}

\section{RESUMEN}

Con el crecimiento acelerado de las resistencias del hormigón a compresión, es necesario considerar modelos de cálculo de las estructuras rigurosos. Las normas de cálculo de estructuras de hormigón armado tienen sus modelos matemáticos basados principalmente en ensayos de laboratorio y usualmente son empíricos. Muchos códigos tienen modelos de cálculo de estructuras bastante conservadores y desactualizados, porque resulta complejo estudiar todas las variables que influyen en el comportamiento de un elemento sometido a esfuerzos cortantes combinados con otros tipos de esfuerzos.

Este trabajo está basado en investigaciones de Collins [1]; donde propuso la Teoría del Campo de Compresión (TCC), más adelante Vecchio y Collins [2] desarrollaron la Teoría del Campo de Compresión Modificada (TCCM) y años más tarde Bentz, Vecchio y Collins [3] presentaron la Teoría del Campo de Compresión Modificada Simplificada (TCCMS), siendo este último un poco menos riguroso pero más simple de ser aplicado.

En este artículo se proponen modelos basados en la TCCM y TCCMS y se muestra resultados de ejemplos aplicando estos modelos comparándolos con las normas ACI 318-11 y CAN23.3-04. En el cálculo de la armadura transversal, la variación entre la TCCM y las normas ACI 318-11 y CAN23.3-04 fue de 33\% y 64\% respectivamente y la variación entre la TCCMS y las normas ACI 318-11 y CAN23.3-04 fue de 2\% y 25\% respectivamente.

Palabras Clave: Resistencia, Cortante, Hormigón Armado. 